[0212-7199 (2008) 25: 3; pp 122-124] ANALES DE MEDICINA INTERNA Copyright (C) 2008 ARAN EDICIONES, S.L.

AN. MED. INTERNA (Madrid) Vol. 25, N. ${ }^{\circ} 3$, pp. 122-124, 2008

\title{
Síndrome overlap hepatitis autoinmune-cirrosis biliar primaria: a propósito de un caso
}

\author{
N. MALLO-GONZÁLEZ, R. LÓPEZ-RODRÍGUEZ, J. CAMPOS-FRANCO, \\ I. ABDULKADER ${ }^{1}$, E. OTERO-ANTÓN, M. R. ALENDE-SIXTO \\ Servicios de Medicina Interna y ${ }^{1}$ Anatomía Patológica. Complejo Hospitalario \\ Universitario de Santiago de Compostela. Santiago de Compostela. A Coruña
}

THE AUTOINMUNE HEPATITIS-PRIMARY BILIARY CIRRHOSIS OVERLAP SYNDROME: A CASE REPORT

\begin{abstract}
RESUMEN
El síndrome overlap hepatitis autoinmune- cirrosis biliar primaria es una entidad caracterizada por manifestaciones clínicas, analíticas, inmunológicas e histológicas de ambas entidades. Presentamos el caso de una mujer de 26 años con una hepatitis aguda grave que cumple los criterios diagnósticos del síndrome de superposición y que respondió de forma satisfactoria al tratamiento con corticoides orales.
\end{abstract}

PALABRAS CLAVE: Síndrome overlap. Síndrome de superposición. Hepatitis autoinmune. Cirrosis biliar primaria.

\begin{abstract}
The autoinmune hepatitis-primary biliary cirrhosis overlap syndrome is an entity characterized by clinical, analytical, immunological and histological manifestations of both entities. We present the case of a 26-year-old woman with a serious acute hepatitis that fulfills the diagnostic criteria of the overlap syndrome and that showed a satisfactory response to oral corticoid therapy.
\end{abstract}

KEY WORDS: Overlap syndrome. Autoinmune hepatitis. Primary biliary cirrosis.

Mallo-González N, López-Rodríguez, R, Campos-Franco J, Abulkader I, Otero-Antón E, Alende-Sixto MR. Síndrome overlap hepatitis autoinmune-cirrosis biliar primaria: a propósito de un caso. An Med Interna (Madrid) 2008; 25: 122-124.

\section{INTRODUCCIÓN}

La hepatitis autoinmune (HAI) y la cirrosis biliar primaria (CBP) son dos hepatopatías crónicas de etiología desconocida y probable etiología inmune cuyo diagnóstico definitivo se realiza de forma concluyente en el $92 \%$ de los casos $(1,2)$. En el resto de los casos presentan características compartidas como pueden ser el patrón bioquímico, la positividad de los anticuerpos y/o la presencia de colangitis en el estudio histológico (3-5). El síndrome overlap HAI-CBP se caracteriza por la superposición de los hallazgos clínicos, analíticos, inmunológicos y/o histológicos de la hepatitis autoinmune y de cirrosis biliar primaria $(3,5-7)$. La patogenia y tratamiento no están completamente definidos en el momento actual, sin embargo parece claro que la respuesta al tratamiento y el pronóstico se encuentra estrechamente relacionado con la entidad que predomina en el síndrome (3,5,8-11).

\section{CASO APORTADO}

Mujer de 26 años de edad sin antecedentes de transfusiones sanguíneas, ingesta de alcohol o exposición a tóxicos y a tratamiento, desde hacía dos años, con anticonceptivos orales. Acudió a Urgen- cias por un cuadro de una semana de evolución de dolor en hipocondrio derecho que se acompaña de astenia, náuseas, coluria, acolia, y febrícula. En la exploración física sólo destacaba la presencia de ictericia cutaneomucosa y un reborde hepático doloroso a la palpación a $4 \mathrm{~cm}$ de la arcada costal. En la analítica de sangre se objetivó una hepatitis aguda grave: aspartato-aminotransferasa (GOT) $4.449 \mathrm{U} / 1$ $(\mathrm{n}=<25)$, alanin-aminotransferasa (GPT) $5.049 \mathrm{U} / \mathrm{l}(\mathrm{n}=<29)$, gammaglutamilaminotransferasa (GGTP) $47 \mathrm{U} / \mathrm{L}(\mathrm{N}: 5-38)$, fosfatasas alcalinas $308 \mathrm{UI} / \mathrm{l}(\mathrm{n}=65-195)$, bilirrubina total 45,3 mg/dl $(\mathrm{N}:<$ $1,20)$, bilirrubina directa $40,9 \mathrm{mg} / \mathrm{dl}(\mathrm{n}=<0,40)$, albúmina $3,5 \mathrm{~g} / \mathrm{dL}$. En el estudio de la coagulación presentaba un tiempo de protrombina del $64 \%$ y un factor $\mathrm{V}$ del $233 \%$. El proteinograma demostró unas proteínas totales de $6,1 \mathrm{~g} / \mathrm{dl}(\mathrm{n}=6,5-8,3)$ con unas gammaglobulinas de $1,7 \mathrm{~g} / \mathrm{dl}$, unos niveles normales de IgG y ligera elevación de IgM $287 \mathrm{mg} / \mathrm{dl}(\mathrm{n}=45-145)$. Las determinaciones serológicas de IgM anti-VHA, anti-VHC, HBsAg, IgM e IgG (o anticuerpos totales) anti-HBc y anticuerpos anti-VIH1/2 fueron todas ellas negativas, mientras que las de VHS, CMV y VEB mostraron un patrón compatible con infección pasada. El estudio inmunológico fue positivo para los anticuerpos antimitocondriales (AMA) con un título superior a 1/1.280, con M2 positivos, mientras que los anticuerpos antinucleares (ANA), anticuerpos anti-músculo liso (ASMA), anticuerpos antimicrosomales hepatorrenales (LKM), anticuerpos anticitosol hepático (LC1) y anticuerpo antiantígeno soluble hepático (SLA) resultaron todos negativos. En los estudios de imagen presentaba una hepatomegalia homogénea con refuerzo portal y granulomas esplénicos cal-

Trabajo aceptado: 22 de octubre de 2007

Correspondencia: Nieves Mallo González. Hospital Clínico Universitario. C/ A Choupana, s/n. 15706 Santiago de Compostela. e-mail: nebesmg@ gmail.com 
cificados. El estudio histológico fue informado lesiones necroinflamatorias a nivel periportal y lobulillar con formación de rosetas e infiltración portal por células plasmáticas, compatible con hepatitis aguda autoinmune. Se inició tratamiento con metilprednisolona a dosis de $1 \mathrm{mg} / \mathrm{kg} / \mathrm{día}$, con resolución completa del cuadro clínico y normalización de los parámetros analíticos al mes de inicio de tratamiento.

\section{DISCUSIÓN}

La hepatitis autoinmune (HAI) es una entidad definida por unos criterios diagnósticos bien establecidos, sin embargo el diagnóstico diferencial con otras enfermedades hepáticas de base inmunológica, en ocasiones, es difícil $(5,6,12)$. De hecho en aproximadamente el $30 \%$ de los casos existen datos que se superponen con otras hepatopatías autoinmunes. Así determinados hallazgos típicos de HAI, particularmente la elevación sérica de IgG, la positividad de ciertos autoanticuerpos y la hepatitis de interfase pueden presentarse en otras enfermedades hepáticas, lo que se conoce como síndrome superposición $(3,6,12,14)$. El diagnóstico de HAI es especialmente complejo en los casos que presentan manifestaciones de cirrosis biliar primaria $(\mathrm{CBP})$, probablemente porque se trate de dos extremos opuestos del espectro de una misma enfermedad hepática autoinmune $(5,8,15)$. Concretamente el $8 \%$ de los casos de hepatitis autoinmune presentan características clínicas, bioquímicas, inmunológicas o histológicas compatibles con $\mathrm{CBP}$ y hasta el $9 \%$ de los pacientes con CBP presentan manifestaciones de HAI, lo que se denomina síndrome overlap CBPHAI $(3,8,9,16)$. La identificación de estos casos es importante ya que su diagnóstico conlleva diferencias pronósticas y tiene importantes implicaciones respecto a la estrategia terapéutica.

El síndrome overlap CBP-HAI afecta sobre todo a mujeres, con una media de edad de 50 años. Las manifestaciones clínicas son inespecíficas, la bioquímica hepática muestra un patrón mixto con presencia de citolisis y colestasis, mientras que en el estudio histológico se objetiva la coexistencia de hallazgos de colangitis con hepatitis de interfase $(3,8)$.

No existen unos criterios diagnósticos consensuados para el síndrome superposición CBP-HAI. La mayoría de los autores utilizan los establecidos por Chazouillères y cols., que para HAI son: a) elevación de GPT sérica al menos 5 veces el límite superior de la normalidad; b) IgG al menos 2 veces el límite superior de la normalidad y/o positividad para anticuerpos ASMA; y c) histología compatible con hepatitis de interfase moderada a severa. Los criterios de CBP comprenden: a) FA al menos 2 veces el límite superior al valor normal y/o GGT al menos 5 veces mayor al límite superior de la normalidad; b) presencia de AMA; y c) lesión de los conductos biliares en el estudio histológico. Siendo preciso para el diagnóstico que se cumplan al menos 2 de los 3 criterios de cada una de las entidades (8).
Sin embargo Ben-Ari y Czaja (4) consideran que los criterios de Chazouillères y cols. (8) se establecieron de forma arbitraria y no tienen una sensibilidad demostrada, por lo que proponen que el diagnóstico de síndrome de superposición CBP-HAI se realice en aquellos pacientes que presenten una clasificación de probable en el sistema de puntuación del Grupo Internacional de Hepatitis Autoinmune (12), asociado a la positividad de los AMA y la presencia de colangitis en el estudio histológico. En este sentido, Yamamoto et cols. (17) proponen un sistema de puntuación para el diagnóstico de la CBP similar al del Grupo Internacional de Hepatitis Autoinmune (12), y comprueban que de todos sus casos con síndrome de superposición presentan puntuaciones elevadas en ambos sistemas de puntuación.

Los AMA son positivos en cerca del $20 \%$ de los casos de hepatitis autoinmune, de los cuales el $88 \%$ presentan títulos iguales o inferiores a 1:160, por lo que títulos bajos de AMA no se considera que tenga relevancia diagnóstica (1). Sin embargo los AMA-M2 están presentes en menos del 8\% de los casos de HAI y pueden ser considerados patognomónicos de CBP $(2,18)$. Por lo que en los pacientes que tienen títulos elevados de AMA-M2 resulta una contradicción establecer el diagnóstico de HAI. Así, la ausencia de alteraciones histológicas de CBP se explicaría porque se encuentran en fases iniciales del síndrome de superposición y/o un error en la biopsia hepática $(3,12)$. De hecho, en las fases iniciales de la CBP las alteraciones histológicas pueden ser parcheadas y focales (13).

No existe un tratamiento de elección y se suele tratar según el componente que predomine en el síndrome overlap CBP-HAI $(3,5,8-11)$. Los casos en que predomina HAI (niveles altos de GPT, FA $<2$ veces el límite normal, moderada a severa hepatitis de interfase y puntuación $\geq 10$ en score de HAI) generalmente responden a corticoides (3,8-11). Mientras que cuando el componente principal del síndrome es la CBP (FA $\geq 2$ veces el límite normal, GGT $\geq 5$ veces el límite normal y lesión ductal) el tratamiento utilizado es la asociación de corticoides y ácido ursodesoxicólico (8).

Nuestra paciente tenía una puntuación de probable en el sistema de puntuación del Grupo Internacional de Hepatitis Autoinmune (12), títulos muy elevados de AMA-M2 y una puntuación de probable en el sistema de puntuación de CBP (17), por lo que consideramos que presentaba un síndrome overlap HAI-CBP con predominio de HAI. Se instauró tratamiento con corticoides, respondiendo favorablemente, con resolución del brote y normalización de los parámetros analíticos.

En nuestra opinión ante la ausencia de criterios validados para el síndrome de superposición HAI-CBP es necesario la comunicación de aquellos casos clínicos compatibles a fin de establecer unos criterios diagnósticos consensuados que abarquen todo el espectro de la enfermedad y establecer las estrategias terapéuticas más favorables.

\section{Bibliografía}

1. Kenny RP, Czaja AJ, Ludwig J, Dickson ER. Frecuency and significance of antimitochondrial antibodies in severe chronic active hepatitis. Dig Dis Sci 1986; 31: 705-11.

2. Czaja AJ, Carpenter HA, Manns MP. Antibodies to soluble liver antigen, P450IID6, and mitocondrial complexes in chronic hepatitis. Gastroenterology $1993 ; 105: 1522-8$.

3. CZAJA AJ. The variant forms of autoinmune hepatitis. Ann Intern Med 1996; 125: 588-98.

4. Ben-Ari Z, Czaja AJ. Autoinmune hepatitis and its variant syndromes. Gut 2001; 49: 589-94.

5. Heathcote J. Variant syndromes of autoimmune hepatitis. Clin Liver Dis 2002; 6: 669-84. 
6. Strassburg CP. Autoimmune liver diseases and their overlap syndromes. Schweiz Rundsch Med Prax 2006; 95: 1363-81.

7. Beuers U. Hepatic overlap syndrome. J Hepatol 2005; 42: S93-S99.

8. Chazouilleres O, Wendum D, Serfaty L, Montembault S, Rosmorduc O, Poupon R. Primary biliary cirrhosis-autoinmune hepatitis overlap syndrome: Clinical features and response to therapy. Hepatology 1998; 28 : 296-301.

9. Lohse AW, Meyer Zum Büschenfelde KH, Franz B, Kanzler S, Gerken G, Dienes HP. Caracterization of the overlap syndrome of primary biliary cirrhosis (PBC) and autoinmune hepatitis: evidence for it being a hepatitic form of PBC in genetically susceptible individuals. Hepatology 1999; 29: 1078-84.

10. Czaja AJ. Frecuency and nature of the variant syndromes of autoinmune liver disease. Hepatology 1998; 28: 360-5.

11. Kenny RP, Czaja AJ, Ludwig J, Dickson ER. Frecuency and significance of antimitochondrial antibodies in severe chronic active hepatitis. Dig Dis Sci 1986; 31: 705-11.

12. Álvarez F, Berg PA, Bianchi FB, Bianchi L, Burroughs AK, Cancado EL, et al. International autoinmune hepatitis group report: Review of criteria for diagnosis of autoinmune hepatitis. J Hepatol 1999; 31: 929-38.

13. Nishio A, Keeffe EB, Ishibashi H, Gershwin EM. Med Sci Monit 2000; 6: 181-93.

14. Talwalkar JA, Keach JC, Angulo P, Lindor KD. Overlap of autoinmune hepatitis and primary biliary cirrhosis: An evaluation of a modified scoring system. Am J Gastroenterol 2002; 97: 1191-7.

15. Suzuki Y, Arase Y, Ikeda K, Saitoh S, Tsubota A, Suzuki F, et al. Clinical and pathological characteristics of the autoinmune hepatitis and primary biliary cirrhosis overlap syndrome. J Gastroenterol Hepatol 2004; 19: 699-706.

16. Goodman ZD, McNally PR, Davis DR, Ishak KG. Autoinmune cholangitis: A variant of primary biliary cirrhosis. Clinico-pathologic and serologic correlation in 200 cases. Dig Dis Sci 1995; 40: 1232-42.

17. Yamamoto K, Terada R, Okamoto R, Hiasa Y, Aabe M, Onji M, et al. A scoring system for primary biliary cirrhosis and its application for variant forms of autoinmune liver disease. J Gastroenterol 2003; 38: 52-9.

18. Metcalf J, Mitchison HC, Palmer JM. Natural history of early primary biliary cirrhosis. Lancet 1996; 348: 1399-402. 